\title{
Wastewater-based epidemiology and group privacy: the elephant in the sewer?
}

Rachel M. Kwiatkowska; ${ }^{\star a, b}$ Anouk Ruhaak; ${ }^{c}$ Barbara Kasprzyk-Hordern; ${ }^{\text {d }}$ Francis Hassard; ${ }^{\mathrm{e}}$

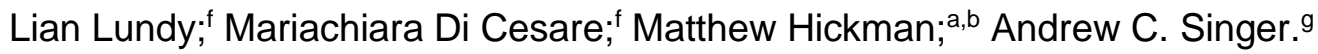
a. Population Health Sciences, University of Bristol Medical School, BS8 2PS, UK
b. NIHR Health Protection Research Unit in Behavioural Science and Evaluation, University of Bristol, BS8 2PS, UK
c. Mozilla Foundation, 10997 Berlin, Germany
d. Department of Chemistry, University of Bath, BA2 7AY, UK
e. Cranfield Water Science Institute, Cranfield University, Bedford, MK43 OAL, UK
f. Department of Natural Sciences, Middlesex University, NW4 4BT, UK
g. UK Centre for Ecology and Hydrology, Wallingford, OX10 8BB, UK

*Email: rachel.kwiatkowska@bristol.ac.uk

Keywords: wastewater surveillance, ethics, wastewater, epidemiology

The utility of wastewater surveillance or wastewater-based epidemiology (WBE) is well established: most notably in the eradication of poliovirus and more recently in mapping trends in SARS-CoV-2 infections during the COVID-19 pandemic. ${ }^{1-4}$ WBE use-cases range from largescale analysis of municipal sewage for illicit drugs and their metabolites to 'near-source tracking' (NST) of the SARS-CoV-2 virus at a building level. ${ }^{5-7}$ Considerable progress has been made on addressing uncertainty in the analysis and interpretation of WBE-derived data ${ }^{8,9}$ and developing ethical guidelines to promote good governance of this data. ${ }^{10-12}$ Nonetheless, there remain concerns around the ethics of WBE. ${ }^{13}$ Why is this?

Wastewater is generally categorised as an environmental matrix, and as such, WBE-derived data are collected without informed consent from 'donors'. As with any environmental monitoring, this can be justified if there is a legitimate public health purpose, such as management of an impending or on-going public or environmental health threat; the means of data collection are proportionate to that purpose; and the data are effectively used to protect the health of the wider population, not just those under surveillance. ${ }^{14}$ The case for implementing WBE is strengthened by elimination of biases associated with self-reported drug use or selfreferral to mass testing for infectious disease and because, being non-invasive, it is inclusive of people who might not otherwise get tested. ${ }^{15}$ WBE is also considered cost-effective for large scale population surveillance, ${ }^{16}$ and 'low risk' with respect to individual privacy and civil liberties. ${ }^{17,18}$

Some have raised questions around ownership of waste products and risks to individual privacy, ${ }^{13,19}$ but we are not aware of Personal Identifiable Information (PII) having been compromised by WBE. As with other surveillance tools, there is, however, a potential risk of harm at the local level, due to social stigma associated with wastewater analysis. For instance, detection of infection markers could inadvertently label certain ethnic groups or marginalised 
communities as 'diseased', fueling existing prejudices. ${ }^{13,20-23}$ WBE must therefore safeguard the rights and liberties of geographically-defined communities, or categories of people. These groups can be identified via Demographic Identifiable Information (DII) - a term referred to by Raymond (2017) as: "either individual or aggregated data points that allow inferences to be drawn that enable the classification, identification and/or tracking of both names and/or unnamed individuals, groups of individuals and/or multiple groups of individuals according to ethnicity, economic class, religion, gender, age, health condition, location, occupation, and/or demographically defining factors." 24

Group privacy, then, is the elephant in the sewer.

Regulations such as the European General Data Protection Regulation (GDPR) ${ }^{25}$ can help manage the ethical pitfalls inherent in public health surveillance, however, they only apply to data that is relatable to an individual - which wastewater is not (at least without considerable additional effort and cost). For the same reason, medical research ethics approvals are not required for WBE. ${ }^{26}$ Ethical regulation of WBE therefore rests with the contributing institutions, ${ }^{10,13,27}$ and whilst ethics boards are experienced in dealing with complex bioethical issues they may not fully consider group privacy factors. ${ }^{28}$ Furthermore, the onus is on wastewater practitioners to define what is in the public's best interests, which might not be appropriate if they lack awareness of public health principles ${ }^{13}$ or the fiduciary responsibility and accountability mechanisms associated with public sector research and surveillance. ${ }^{20}$ Finally, and importantly, the determination of 'the public's best interest' is usually made without input from the groups who might suffer unintended consequences.

Despite a reassuring lack of data governance breaches, the possibility of fallout as a result of processing DII jeopardises the future of WBE as a valuable surveillance tool. In response to this risk, as well as the limitations of existing data protection frameworks and regulations in the face of a public health emergency, ${ }^{29}$ we propose that now is the time to pro-actively strengthen the public health toolkit.

There is a strong argument for involving communities and stakeholders in co-development and regulation of WBE programmes ${ }^{10-12}$ to address group privacy issues particularly within nearsource WBE initiatives. ${ }^{30}$ Organisations such as the Ada Lovelace Institute and Mozilla are pioneering participatory stewardship frameworks, approaches to collective consent, and using citizen councils to rule on the collection and use of digital and biometric data. ${ }^{31-35}$ We find other examples in the cooperative space, for instance Driver's Coop in the US, whereby decisions are made collectively about what data are collected and how they are used. ${ }^{36}$ This work provides a useful foundation for strengthening DII data governance and promoting ethically-responsible WBE data collection; we define this as:

1. Having a clear and measurable purpose, with demonstrable impact on public health.

2. Having a thorough understanding of the benefits, risks and harms.

3. Implementing robust and ongoing oversight to ensure Criteria 1 and 2 are met. 
Under a participatory stewardship framework, those described by the data or affected by its collection and use (the data subjects) can ensure all three criteria are met. Specifically, they should have a say in:

- designing the WBE programme

- defining 'public good/ best interests', and

- deciding on rules for the collection, accessibility and use of this data.

Data subjects should ideally also be empowered to:

- monitor compliance with rules on data use,

- hold those who collect and use the data accountable to these rules, and

- access clear and cost-effective ways to register and negotiate any conflict.

Active participation by data subjects, together with robust accountability mechanisms that enforce participants' decisions, will help ensure that WBE programmes benefit the publics they aim to serve - assuming a balance can be struck on the time, bureaucracy, and financial outlays associated with this approach. We therefore urge practitioners to develop participatory systems and best practices for protecting DII, and in doing so hope that WBE can lead the way in responsible public health data collection.

\section{Conflicts of interest declaration}

The authors declare no conflicts of interest.

\section{Funding}

RK is funded through the Wellcome GW4 Clinical Academic Training programme [203918].

\section{References}

1. Asghar, H. et al. Environmental surveillance for polioviruses in the Global Polio Eradication Initiative. J. Infect. Dis. 210 Suppl 1, S294-303 (2014).

2. O'Reilly, K. M., Allen, D. J., Fine, P. \& Asghar, H. The challenges of informative wastewater sampling for SARS-CoV-2 must be met: lessons from polio eradication. Lancet Microbe 1, e189-e190 (2020).

3. Peccia, J. et al. Measurement of SARS-CoV-2 RNA in wastewater tracks community infection dynamics. Nat. Biotechnol. 38, 1164-1167 (2020).

4. La Rosa, G. et al. First detection of SARS-CoV-2 in untreated wastewaters in Italy. Sci. Total Environ. 736, 139652 (2020). 
5. European Monitoring Centre for Drugs and Drug Addiction, E. Wastewater analysis and drugs - a European multi-city study. www.emcdda.europa.eu https://www.emcdda.europa.eu/publications/html/pods/waste-water-analysis_en (2021).

6. Jin, H. et al. Estimation of the psychoactive substances consumption within 12 wastewater treatment plants service areas in a certain city of Guangxi, China applying wastewater-based epidemiology. Sci. Total Environ. 778, 146370 (2021).

7. Davó, L. et al. Early detection of SARS-CoV-2 infection cases or outbreaks at nursing homes by targeted wastewater tracking. Clin. Microbiol. Infect. 27, 1061-1063 (2021).

8. Wade, M. J. et al. Understanding and managing uncertainty and variability for wastewater monitoring beyond the pandemic: Lessons learned from the United Kingdom national COVID-19 surveillance programmes. J. Hazard. Mater. 424, 127456 (2021).

9. Kevill, J. L. et al. A comparison of precipitation and filtration-based SARS-CoV-2 recovery methods and the influence of temperature, turbidity, and surfactant load in urban wastewater. Sci. Total Environ. 151916 (2021) doi:10.1016/j.scitotenv.2021.151916.

10. Sewage Analysis CORe group - Europe (SCORE) et al. Ethical research guidelines for wastewater-based epidemiology and related fields Version 1. https://scorecost.eu/ethical-guidelines-for-wbe/ (2015).

11. Hrudey, S. E. et al. Ethics Guidance for Environmental Scientists Engaged in Surveillance of Wastewater for SARS-CoV-2. Environ. Sci. Technol. 55, 8484-8491 (2021).

12. Canadian Coalition on Wastewater-related COVID-19 Research. Ethics and communications guidance for wastewater surveillance to inform public health decisionmaking about COVID-19. https://cwn-rce.ca/wp-content/uploads/COVID19-WastewaterCoalition-Ethics-and-Communications-Guidance-v4-Sept-2020.pdf (2020). 
13. Coffman, M. M. et al. Preventing scientific and ethical misuse of wastewater surveillance data. Environ. Sci. Technol. 55, 11473-11475 (2021).

14. Klingler, C. et al. Ethical issues in public health surveillance: a systematic qualitative review. BMC Public Health 17, 295 (2017).

15. Hendriksen, R. S. et al. Global monitoring of antimicrobial resistance based on metagenomics analyses of urban sewage. Nat. Commun. 10, 1124 (2019).

16. Hart, O. E. \& Halden, R. U. Computational analysis of SARS-CoV-2/COVID-19 surveillance by wastewater-based epidemiology locally and globally: Feasibility, economy, opportunities and challenges. Sci. Total Environ. 730, 138875 (2020).

17. Prichard, J., Hall, W., de Voogt, P. \& Zuccato, E. Sewage epidemiology and illicit drug research: the development of ethical research guidelines. Sci. Total Environ. 472, 550555 (2014).

18. Hall, W. et al. An analysis of ethical issues in using wastewater analysis to monitor illicit drug use. Addiction 107, 1767-1773 (2012).

19. Gable, L., Ram, N. \& Ram, J. L. Legal and ethical implications of wastewater monitoring of SARS-CoV-2 for COVID-19 surveillance. J. Law Biosci. 7, Isaa039 (2020).

20. Keshaviah, A., Hu, X. C. \& Henry, M. Developing a Flexible National Wastewater Surveillance System for COVID-19 and Beyond. Environ. Health Perspect. 129, 45002 (2021).

21. Polo, D. et al. Making waves: Wastewater-based epidemiology for COVID-19 approaches and challenges for surveillance and prediction. Water Res. 186, 116404 (2020). 
22. Roberto, K. J., Johnson, A. F. \& Rauhaus, B. M. Stigmatization and prejudice during the COVID-19 pandemic. Administrative Theory \& Praxis 1-15 (2020)

doi:10.1080/10841806.2020.1782128.

23. A guide to preventing and addressing social stigma associated with COVID-19. https://www.who.int/publications/m/item/a-guide-to-preventing-and-addressing-socialstigma-associated-with-covid-19.

24. Raymond, N. A. Beyond "Do No Harm" and Individual Consent: Reckoning with the Emerging Ethical Challenges of Civil Society's Use of Data. in Group Privacy (eds. Taylor, L., Floridi, L. \& van der Sloot, B.) 67-82 (Springer International Publishing, 2017). doi:10.1007/978-3-319-46608-8_4.

25. UK Information Commissioner's Office. Guide to the UK General Data Protection Regulation (UK GDPR). https://ico.org.uk/for-organisations/guide-to-dataprotection/guide-to-the-general-data-protection-regulation-gdpr/.

26. NHS Health Research Authority, UKRI \& Medical Research Council. Do I need NHS Ethics approval? http://www.hra-decisiontools.org.uk/ethics/.

27. van Dyken, E. et al. Monitoring substance use in prisons: Assessing the potential value of wastewater analysis. Sci. Justice 54, 338-345 (2014).

28. Lancaster, K., Rhodes, T., valentine, kylie \& Ritter, A. A 'promising tool'? A critical review of the social and ethico-political effects of wastewater analysis in the context of illicit drug epidemiology and drug policy. Current Opinion in Environmental Science \& Health 9, 8590 (2019).

29. COVID-19 response and data protection law in the EU and US. https://iapp.org/news/a/covid-19-response-and-data-protection-law-in-the-eu-and-us/. 
30. Ethical considerations in the use of geospatial data for research and statistics - UK Statistics Authority. https://uksa.statisticsauthority.gov.uk/publication/ethicalconsiderations-in-the-use-of-geospatial-data-for-research-and-statistics/pages/3/.

31. Mozilla Foundation - When One Affects Many: The Case For Collective Consent. https://foundation.mozilla.org/en/blog/when-one-affects-many-case-collective-consent/.

32. Delacroix, S. \& Lawrence, N. D. Bottom-up data Trusts: disturbing the 'one size fits all' approach to data governance. International Data Privacy Law (2019) doi:10.1093/idpl/ipz014.

33. Arnstein, S. R. A Ladder Of Citizen Participation. J. Am. Inst. Plann. 35, 216-224 (1969).

34. The Citizens' Biometrics Council | Ada Lovelace Institute. https://www.adalovelaceinstitute.org/project/citizens-biometrics-council/.

35. Participatory data stewardship | Ada Lovelace Institute. https://www.adalovelaceinstitute.org/report/participatory-data-stewardship/.

36. The Drivers Cooperative. https://drivers.coop/. 